\title{
Efficient estimation of high-dimensional multivariate normal copula models with discrete spatial responses
}

\author{
Aristidis K. Nikoloulopoulos*
}

\begin{abstract}
The distributional transform (DT) is amongst the computational methods used for estimation of high-dimensional multivariate normal copula models with discrete responses. Its advantage is that the likelihood can be derived conveniently under the theory for copula models with continuous margins, but there has not been a clear analysis of the adequacy of this method. We investigate the small-sample and asymptotic efficiency of the method for estimating high-dimensional multivariate normal copula models with univariate Bernoulli, Poisson, and negative binomial margins, and show that the DT approximation leads to biased estimates when there is more discretization. For a high-dimensional discrete response, we implement a maximum simulated likelihood method, which is based on evaluating the multidimensional integrals of the likelihood with randomized quasi Monte Carlo methods. Efficiency calculations show that our method is nearly as efficient as maximum likelihood for fully specified highdimensional multivariate normal copula models. Both methods are illustrated with spatially aggregated count data sets, and it is shown that there is a substantial gain on efficiency via the maximum simulated likelihood method.
\end{abstract}

Keywords: Areal data; Distributional transform, Generalized quantile transform; Rectangle probabilities; Simulated likelihood; Spatially aggregated data.

${ }^{*}$ A. Nikoloulopoulos@uea.ac.uk, School of Computing Sciences, University of East Anglia, Norwich NR4 7TJ, UK 


\section{Introduction}

There are classical statistical models in the literature for regression and prediction with spatial continuous data for which parameter estimation and inference are straightforward. These models are based on the multivariate normal (MVN) distribution, applied to a possibly transformed response (for a thorough review see Diggle and Ribeiro (2007)). But applications of these models are invalid for discrete spatial categorical and count response data. These data occur in several disciplines such as epidemiology, ecology, agriculture, to name just a few. Flexible models for such data are not widely available and usually hard to fit due to the fact that available multivariate discrete distributions can have only certain properties, as for example they provide limited dependence or they can have marginal distributions of a given form. Most of the existing literature is concerned with the generalized linear mixed model proposed by Diggle et al. (1998). This model was initially proposed for the analysis of correlated count data by Aitchinson and Ho (1989). Limitations of this model are that the marginal distributions belong to a specific parametric family, and, because the correlation structure come from a continuous multivariate mixing distribution, the possible choices are very limited. Thus, it seems that there is a lack of flexible models appropriate for spatial discrete data or flexible marginal choices.

In this paper, we use copulas (distributions with uniform margins on the unit interval) to overcome this problem. The power of copulas for dependence modelling is due to the dependence structure being considered separate from the univariate margins; see, for example, Section 1.6 of Joe (1997). A discrete regression spatial model through copulas is not new. A copula approach was recently proposed and studied by Madsen (2009), Kazianka and Pilz (2010), Kazianka (2013) and Hughes (2014) who explored the use of

MVN copulas to describe the distribution of geostatistical and spatially aggregated data, respectively.

Our empirical experience is that the MVN copula model with discrete margins provides the best or nearly the best fit. However, implementation of the MVN copula for discrete data is possible, but not easy, because the MVN distribution as a latent model for 
discrete response requires rectangle probabilities based on multidimensional integrations (Nikoloulopoulos et al., 2011). Song (2007), influencing other authors (e.g. Kazianka (2013), Hughes (2014)), acknowledged that the probability mass function (pmf) can be obtained as a finite difference of the copula cumulative distribution function (cdf). Generally speaking, this is an imprecise statement, since calculating the finite difference among $2^{d}$ (where $d$ is the dimension) numerically computed orthant probabilities may result in negative values. The pmf can be alternatively obtained by computing a MVN rectangle probability. The randomized quasi Monte Carlo methods proposed by Genz (1992) and Genz and Bretz (2002) can be used for that purpose. If one computes the rectangle MVN probabilities via simulation based on the methods in Genz (1992) and Genz and Bretz (2002), then one is using a simulated likelihood method. Nikoloulopoulos (2013b) has studied the asymptotic and small-sample efficiency of this simulated likelihood method and has shown it is as good as maximum likelihood for dimension 10 or lower.

Madsen (2009) and Madsen and Fang (2011) proposed a different simulated likelihood method to "approximate" the likelihood, by using the continuous extension (CE) of a discrete random variable developed in Denuit and Lambert (2005) and applied the method to geostatistical and longitudinal discrete data, respectively. Shi and Valdez (2014a,b) also used the simulated likelihood method based on the CE for longitudinal and multivariate insurance claim counts.

Kazianka and Pilz (2010) and Kazianka (2013) proposed and studied respectively a fast surrogate likelihood method, by approximating the rectangle probability with a copula density using the distributional transform (DT); see Ferguson (1967) for an early appearance. Both the simulated likelihood method based on the $\mathrm{CE}$ and the surrogate likelihood method based on the DT have the advantage that the likelihood can be derived conveniently under the theory for copula models with continuous margins.

Hughes (2014) recommended the simulated likelihood method based on the CE and the surrogate likelihood method based on the DT along with the composite likelihood (CL) method (Varin, 2008) for modelling spatially aggregated discrete (areal) data and used the 
simulated likelihood method based on the CE to "judge" the DT and CL approaches. It came as somewhat of a surprise to us, because (a) asymptotic and small-sample efficiency calculations in Nikoloulopoulos (2013b) have shown that the simulated likelihood method based on the CE is a very inefficient approach; it leads to substantial downward bias for the estimates of the latent correlation and the univariate marginal parameters that they are not regression coefficients for fully specified multivariate normal copula-based models, and, (b) CL methods are well established as an alternative of maximum likelihood when the joint probability is too difficult to compute; they lead to unbiased estimating equations (Varin et al., 2011). Note in passing that they have been studied for copula modelling by Zhao and Joe (2005).

The surrogate likelihood method based on the DT cannot be recommended until its properties have been studied and compared to efficient existing methods. The DT method has been previously used in copula literature, e.g. to prove stochastic ordering results and construct tests of dependence properties in Rüschendorf (1981) and Rüschendorf (2009), respectively. Although its application to copula dependence modelling for discrete data is novel, its asymptotic properties has yet to be established in that context.

The contribution in this paper is (a) to examine thoroughly the accuracy and the adequacy of the surrogate likelihood method based on the DT using asymptotics and smallsample efficiency studies; (b) to study the estimation of the MVN copula model with discrete responses via the simulated likelihood method proposed by Nikoloulopoulos (2013b) in a high-dimensional/spatial context.

The remainder of the paper proceeds as follows. Section 2 has a brief overview for MVN copula models. Likelihood estimation methods are provided in Section 3. Section 4 and Section 5 contain theoretical (asymptotic properties of the estimators) and smallsample efficiency calculations, respectively, to assess the accuracy of the discussed likelihood estimation methods. Section 6 presents applications of the likelihood estimation methods to two areal data sets. In these examples, it turns out that the surrogate likelihood method based on the DT could lead to invalid inference. We conclude with some discussion 
in Section 7.

\section{Overview and relevant background for MVN copula models}

A copula is a multivariate cdf with uniform $U(0,1)$ margins (Joe, 1997, 2014; Nelsen, 2006). If $F$ is a $d$-variate cdf with univariate margins $F_{1}, \ldots, F_{d}$, then Sklar's (1959) theorem implies that there is a copula $C$ such that

$$
F\left(y_{1}, \ldots, y_{d}\right)=C\left(F_{1}\left(y_{1}\right), \ldots, F_{d}\left(y_{d}\right)\right)
$$

The copula is unique if $F_{1}, \ldots, F_{d}$ are continuous, but not if some of the $F_{j}$ have discrete components. If $F$ is continuous and $\left(Y_{1}, \ldots, Y_{d}\right) \sim F$, then the unique copula is the distribution of $\left(U_{1}, \ldots, U_{d}\right)=\left(F_{1}\left(Y_{1}\right), \ldots, F_{d}\left(Y_{d}\right)\right)$ leading to

$$
C\left(u_{1}, \ldots, u_{d}\right)=F\left(F_{1}^{-1}\left(u_{1}\right), \ldots, F_{d}^{-1}\left(u_{d}\right)\right), \quad 0 \leq u_{j} \leq 1, j=1, \ldots, d
$$

where $F_{j}^{-1}$ are inverse cdfs. In particular, if $\Phi_{d}(\cdot ; \mathbf{R})$ is the MVN cdf with correlation matrix

$$
\mathbf{R}=\left(\rho_{j k}: 1 \leq j<k \leq d\right)
$$

and $\mathrm{N}(0,1)$ margins, and $\Phi$ is the univariate standard normal cdf, then the MVN copula is

$$
C\left(u_{1}, \ldots, u_{d} ; \mathbf{R}\right)=\Phi_{d}\left(\Phi^{-1}\left(u_{1}\right), \ldots, \Phi^{-1}\left(u_{d}\right) ; \mathbf{R}\right)
$$

Consider a multivariate discrete regression setup in which $d \geq 2$ dependent discrete random variables $Y_{1}, \ldots, Y_{d}$ are observed together with a vector $\mathbf{x} \in \mathbb{R}^{p}$ of explanatory variables. If $C(\cdot ; \mathbf{R})$ is the MVN copula (or any other parametric family of copulas) and $F_{j}(\cdot ; \nu, \gamma)$, where $\nu=\eta(\mathbf{x} ; \boldsymbol{\beta})$ is a function of $\mathbf{x}$ and the $p$-dimensional regression vector $\boldsymbol{\beta}$, and $\boldsymbol{\gamma}$ is the $r$-dimensional vector of univariate parameters that are not regression coefficients, is a parametric model for the $j$ th univariate margin then

$$
C\left(F_{1}\left(y_{1} ; \nu_{1}, \gamma\right), \ldots, F_{d}\left(y_{d} ; \nu_{d}, \gamma\right) ; \mathbf{R}\right)
$$

is a multivariate parametric model with univariate margins $F_{1}, \ldots, F_{d}$. For copula models, the response vector $\mathbf{Y}=\left(Y_{1}, \ldots, Y_{d}\right)$ can be discrete (Nikoloulopoulos, 2013a; Nikoloulopoulos and Joe, 2013). 


\section{Likelihood estimation methods}

In this section, we discuss the simulated likelihood method proposed by Nikoloulopoulos (2013b) and the surrogate likelihood method based on the DT (Hughes, 2014; Kazianka, 2013; Kazianka and Pilz, 2010). The simulated likelihood method based on the CE (Hughes, 2014; Madsen, 2009; Madsen and Fang, 2011; Shi and Valdez, 2014a,b) is not discussed/used in the sequel since its inefficiency has been shown in Nikoloulopoulos (2013b) and should be avoided for copula dependence modelling with multivariate/longitudinal discrete data.

\subsection{Simulated likelihood}

For a sample of size $n$ with data $\mathbf{y}_{1}, \ldots, \mathbf{y}_{n}$, the joint log-likelihood of a MVN copula model is

$$
\ell(\boldsymbol{\beta}, \boldsymbol{\gamma}, \mathbf{R})=\sum_{i=1}^{n} \log h\left(y_{i 1}, \ldots, y_{i d} ; \boldsymbol{\beta}, \boldsymbol{\gamma}, \mathbf{R}\right),
$$

where $h(\cdot ; \boldsymbol{\beta}, \boldsymbol{\gamma}, \mathbf{R})$ is the joint pmf of the multivariate discrete response vector $\mathbf{Y}$. The pmf can be obtained by computing the following rectangle probability,

$$
\begin{aligned}
h(\mathbf{y} ; \boldsymbol{\beta}, \boldsymbol{\gamma}, \mathbf{R}) & =\operatorname{Pr}\left(Y_{1}=y_{1}, \ldots, Y_{d}=y_{d} ; \mathbf{x}\right) \\
& =\operatorname{Pr}\left(y_{1}-1<Y_{1} \leq y_{1}, \ldots, y_{d}-1<Y_{d} \leq y_{d} ; \mathbf{x}\right) \\
& =\int_{\Phi^{-1}\left[F_{1}\left(y_{1}-1 ; \nu_{1}, \gamma\right)\right]}^{\Phi^{-1}\left[F_{1}\left(y_{1} ; \nu_{1}, \gamma\right)\right]} \cdots \int_{\Phi^{-1}\left[F_{d}\left(y_{d}-1 ; \nu_{d}, \gamma\right)\right]}^{\Phi^{-1}\left[F_{d}\left(y_{d} ; \nu_{d}, \gamma\right)\right]} \phi_{\mathbf{R}}\left(z_{1}, \ldots, z_{d}\right) d z_{1} \ldots d z_{d}
\end{aligned}
$$

where $\phi_{\mathbf{R}}$ denotes the standard MVN density with latent correlation matrix $\mathbf{R}$.

There are several papers in the literature that focus on the computation of the MVN rectangle probabilities. The dominant of the methods is a quasi Monte Carlo method proposed by Genz (1992) and Genz and Bretz (2002). The method achieves error reduction of Monte Carlo methods with variance reduction methods as (a) transforming to a bounded integrand, (b) using antithetic variates, and (c) using a randomized quasi Monte Carlo method. The test results in Genz and Bretz $(2002,2009)$ show that the method is very efficient, compared to other methods in the literature. Note in passing that the implementation of the proposed algorithms in Genz and Bretz (2002) is available in the mvtnorm package in R 
(Genz et al., 2012). This advance in computation of MVN probabilities can be used to implement high-dimensional MVN copula models with discrete response data.

Nikoloulopoulos (2013b) proposed a simulated likelihood (hereafter SL) method, where the rectangle MVN probabilities in (2) are computed based on the methods in Genz and Bretz (2002). The estimated parameters can be obtained by maximizing the simulated loglikelihood in (2) over the univariate and copula parameters $(\boldsymbol{\beta}, \boldsymbol{\gamma}, \mathbf{R})$. Since the estimation of the parameters of the MVN copula-based models is obtained using a quasi-Newton routine (Nash, 1990) applied to the log-likelihood in (2), the use of randomized quasi Monte Carlo simulation to four decimal place accuracy for evaluations of integrals works poorly, because numerical derivatives of the log-likelihood with respect to the parameters are not smooth. In order to achieve smoothness, the same set of uniform random variables should be used for every rectangle probability that comes up in the optimization of the SL. The method was initially proposed for the analysis of discrete longitudinal data (Nikoloulopoulos, 2013b). We refer the interested reader to this paper for more details.

\subsection{Surrogate likelihood based on the DT}

Copula models were originally developed for continuous responses where the density is obtained using partial derivatives of the multivariate copula cdf, and hence the numerical calculations are much simpler. Kazianka and Pilz (2010) and Kazianka (2013) proposed and studied respectively a surrogate likelihood method, by approximating the rectangle probability in (3) with a copula density using the DT (Ferguson, 1967).

The surrogate likelihood takes the form

$$
\ell(\boldsymbol{\beta}, \boldsymbol{\gamma}, \mathbf{R}) \approx \sum_{i=1}^{n} \log c\left(v_{i 1}, \ldots, v_{i d} ; \mathbf{R}\right)+\sum_{i=1}^{n} \sum_{j=1}^{d} \log f_{j}\left(y_{i j} ; \nu_{i j}, \boldsymbol{\gamma}\right)
$$

where $v_{j}=0.5\left(F_{j}\left(y_{j} ; \nu_{j}, \gamma\right)+F_{j}\left(y_{j}-1 ; \nu_{j}, \gamma\right)\right)$ and $f_{1}\left(y_{1} ; \nu_{1}, \gamma\right), \ldots, f_{d}\left(y_{d} ; \nu_{d}, \gamma\right)$ are the univariate marginal pmfs. Since the MVN copula has a closed form density

$$
c\left(u_{1}, \ldots, u_{d} ; \mathbf{R}\right)=|\mathbf{R}|^{-1 / 2} \exp \left[\frac{1}{2}\left\{\mathbf{q}^{\top}\left(\mathbf{I}_{d}-\mathbf{R}^{-1}\right) \mathbf{q}\right\}\right]
$$

where $\mathbf{q}=\left(q_{1}, \ldots, q_{d}\right)$ with $q_{j}=\Phi^{-1}\left(u_{j}\right), j=1, \ldots, d$ and $\mathbf{I}_{d}$ is the $d$-dimensional iden- 
tity matrix, the authors avoid the multidimensional integration, and hence the numerical calculations are much simpler and faster. The estimated parameters can be obtained by maximizing the surrogate log-likelihood in (4) over the univariate and copula parameters $(\boldsymbol{\beta}, \boldsymbol{\gamma}, \mathbf{R})$.

\section{Asymptotics}

In this section, we study the asymptotics of the surrogate likelihood method based on the DT (Hughes, 2014; Kazianka, 2013; Kazianka and Pilz, 2010), along with the asymptotics of the SL in Nikoloulopoulos (2013b), and we assess the accuracy based on the limit (as the number of clusters increases to infinity) of the maximum surrogate likelihood estimate (DTMLE) and the maximum SL estimate (MSLE). We restrict ourselves to a MVN copula model with a positive exchangeable dependence structure, i.e., we took $\mathbf{R}$ as $(1-\rho) \mathbf{I}_{d}+\rho \mathbf{J}_{d}$, where $\mathbf{J}_{d}$ is the $d \times d$ matrix of $1 \mathrm{~s}$. For positive exchangeable correlation structures, the $d$-dimensional integrals conveniently reduce to 1-dimensional integrals (Johnson and Kotz, 1972, p. 48). Hence, MVN rectangle probabilities can be quickly computed to a desired accuracy that is $10^{-6}$ or less, because 1-dimensional numerical integrals are computationally easier than higher-dimensional numerical integrals. If one computes the rectangle MVN probabilities in (2) with the 1-dimensional integral method in Johnson and Kotz (1972), then one is using a numerically accurate likelihood method that is valid for any dimension (Nikoloulopoulos, 2013b).

By varying factors such as dimension $d$, the amount of discreteness (binary versus count response), and latent correlation for exchangeable structures, we demonstrate patterns in the asymptotic bias of the DTMLE and MSLE, and assess the performance of the surrogate and simulated likelihood. Note that the performance of the SL method has been already assessed in Nikoloulopoulos (2013b) and it is shown that it is good as maximum likelihood for dimension 10 or lower. For the cases where we compute the probability limit, we will take a constant dimension $d$ that increases. For marginal models we use Bernoulli $(\mu)$, $\operatorname{Poisson}(\mu)$, and negative binomial (NB). For the latter model, we use both the $\operatorname{NB} 1(\mu, \gamma)$ 
and $\operatorname{NB} 2(\mu, \gamma)$ parametrization in Cameron and Trivedi (1998); the NB2 parametrization is that used in Lawless (1987). For ease of exposition, we also consider the case that $\mu$ is common to different univariate margins and does not depend on covariates.

Let the $T$ distinct cases for the discrete response be denoted as

$$
\mathbf{y}^{(1)}, \ldots, \mathbf{y}^{(T)}, \quad \mathbf{y}^{(t)}=\left(y_{1}^{(t)}, \ldots, y_{d}^{(t)}\right), t=1, \ldots, T
$$

In a random sample of size $n$, let the corresponding frequencies be denoted as $n^{(1)}, \ldots, n^{(T)}$ and $p^{(t)}$ be the limit in probability of $n^{(t)} / n$ as $n \rightarrow \infty$. For the SL in (2), we have the limit

$$
n^{-1} \ell(\mu, \gamma, \rho) \rightarrow \sum_{t=1}^{T} p^{(t)} \log h\left(y_{1}^{(t)}, \ldots, y_{d}^{(t)} ; \mu, \gamma, \mathbf{R}\right)
$$

where $h\left(\mathbf{y}^{(t)} ; \mu, \gamma, \mathbf{R}\right)$ is computed using the method in Genz and Bretz (2002). The limit of the MSLE (as $n \rightarrow \infty)$ is the maximum of (5); we denote this limit as $\left(\mu^{S L}, \gamma^{S L}, \rho^{S L}\right)$. Note in passing that the limit of the standard MLE (as $n \rightarrow \infty$ ) is the maximum of (5) where $h\left(\mathbf{y}^{(t)} ; \mu, \gamma, \mathbf{R}\right)$ is computed with the 1-dimensional integral method in Johnson and Kotz (1972). For the surrogate log-likelihood in (4), we have the limit

$$
n^{-1} \ell(\mu, \gamma, \rho) \rightarrow \sum_{t=1}^{T} p^{(t)}\left\{\log c\left(v_{1}^{(t)}, \ldots, v_{d}^{(t)} ; \mathbf{R}\right)+\sum_{j=1}^{d} \log f_{j}\left(y_{j}^{(t)} ; \mu, \gamma\right)\right\}
$$

where $v^{(t)}=0.5\left(F_{j}\left(y^{(t)} ; \mu, \gamma\right)+F_{j}\left(y^{(t)}-1 ; \mu, \gamma\right)\right)$. The limit of the DTMLE $($ as $n \rightarrow \infty)$ is the maximum of (6); we denote this limit as $\left(\mu^{D T}, \gamma^{D T}, \rho^{D T}\right)$.

We will compute these limiting MSLE and DTMLE in a variety of situations to show clearly if the simulated and surrogate likelihood methods are good. By using these limits, we do not need Monte Carlo simulations for comparisons, and we can quickly vary parameter values and see the effects. The $p^{(t)}$ in (5) and (6) are the model based probabilities $h\left(\mathbf{y}^{(t)} ; \mu, \gamma, \mathbf{R}\right)$, and computed with the 1-dimensional integral method in Johnson and Kotz (1972). For a count response, we get a finite number of $\mathbf{y}^{(t)}$ vectors by truncation. The truncation point is chosen to exceed 0.999 for total probabilities.

Representative results are shown in Tables 1 and 2 for $\operatorname{Bernoulli}(\mu)$, and $\operatorname{NB} 2(\mu, \gamma)$ margins, with MSLE results omitted because they were identical with MLE up to three or 


\begin{tabular}{ccccc|ccccc}
\hline$\mu$ & \multicolumn{4}{c|}{$\mu^{D T}$} & $\rho$ & \multicolumn{5}{c}{$\rho^{D T}$} \\
& $d=2$ & $d=3$ & $d=5$ & $d=10$ & & $d=2$ & $d=3$ & $d=5$ & $d=10$ \\
\hline 0.2 & 0.225 & 0.245 & 0.269 & 0.290 & 0.2 & 0.605 & 0.643 & 0.674 & 0.696 \\
0.5 & 0.500 & 0.500 & 0.500 & 0.500 & 0.2 & 0.488 & 0.523 & 0.555 & 0.579 \\
0.8 & 0.775 & 0.755 & 0.731 & 0.710 & 0.2 & 0.605 & 0.643 & 0.674 & 0.696 \\
0.2 & 0.232 & 0.253 & 0.274 & 0.293 & 0.5 & 0.715 & 0.736 & 0.752 & 0.764 \\
0.5 & 0.500 & 0.500 & 0.500 & 0.500 & 0.5 & 0.650 & 0.664 & 0.677 & 0.686 \\
0.8 & 0.768 & 0.747 & 0.726 & 0.707 & 0.5 & 0.715 & 0.736 & 0.752 & 0.764 \\
0.2 & 0.240 & 0.261 & 0.280 & 0.297 & 0.8 & 0.834 & 0.842 & 0.849 & 0.854 \\
0.5 & 0.500 & 0.500 & 0.500 & 0.500 & 0.8 & 0.800 & 0.805 & 0.808 & 0.811 \\
0.8 & 0.760 & 0.739 & 0.720 & 0.703 & 0.8 & 0.834 & 0.842 & 0.849 & 0.854 \\
\hline
\end{tabular}

Table 1: Limiting DTMLE for MVN copula-based models with Bernoulli $(\mu)$ margins.

four decimal places. Therefore, the SL method leads to unbiased estimates. As regard as the surrogate likelihood method, for binary responses there is substantial asymptotic bias for both the univariate and latent correlation parameters. The only case that there is asymptotic unbiasedness for $\mu$ is when $\mu=0.5$. This is due the fact that the individual probabilities have the same size. For non-overdispersed count responses the surrogate likelihood method leads to approximate asymptotic unbiasedness for both the univariate and latent correlation parameters as $\mu$ increases. However, for overdispersed count responses, the method leads to asymptotic bias (decreases as $\mu$ increases or $\gamma$ or $\rho$ or $d$ decreases) for all the univariate parameters and substantial asymptotic upward bias for the latent correlation $\rho$ (decreases as $\rho$ increases).

To sum up, the DT approximation is worse with more discretization (larger individual probabilities). The result should not be in terms of higher mean values as in Kazianka (2013), but in terms of the size of the discrete probabilities. For higher mean values and moderate overdispersion, the probability of any given event will decrease, and as a result, one would be linearly approximating 'smaller steps' in the cdf.

After evaluating the adequacy of the simulated and surrogate log-likelihood on finding the peak (MLE), we evaluate if the curvature (Hessian) is also correct for the cases where the MSLE and DTMLE are correct. To check this, we also computed the negative inverse Hessian $H$ of the limit of the surrogate log-likelihood in (6) and the simulated log- 


\begin{tabular}{ccc|ccc|ccc}
\hline$\mu$ & \multicolumn{2}{c|}{$\mu^{D T}$} & \multicolumn{2}{c|}{$\gamma^{D T}$} & $\rho$ & \multicolumn{3}{c}{$\rho^{D T}$} \\
& $d=2$ & $d=3$ & & $d=2$ & $d=3$ & & $d=2$ & $d=3$ \\
\hline 0.5 & 0.504 & 0.526 & 0.5 & 0.603 & 0.759 & 0.2 & 0.348 & 0.419 \\
1 & 0.994 & 0.992 & 0.5 & 0.530 & 0.567 & 0.2 & 0.259 & 0.283 \\
2 & 1.992 & 1.985 & 0.5 & 0.510 & 0.521 & 0.2 & 0.225 & 0.234 \\
5 & 4.993 & 4.987 & 0.5 & 0.502 & 0.505 & 0.2 & 0.208 & 0.210 \\
10 & 9.995 & 9.991 & 0.5 & 0.501 & 0.501 & 0.2 & 0.203 & 0.204 \\
0.5 & 0.522 & 0.604 & 2 & 2.248 & 2.632 & 0.2 & 0.390 & 0.498 \\
1 & 1.001 & 1.020 & 2 & 2.103 & 2.232 & 0.2 & 0.292 & 0.335 \\
2 & 1.989 & 1.987 & 2 & 2.055 & 2.118 & 0.2 & 0.252 & 0.272 \\
5 & 4.976 & 4.956 & 2 & 2.029 & 2.059 & 0.2 & 0.228 & 0.238 \\
10 & 9.964 & 9.932 & 2 & 2.018 & 2.037 & 0.2 & 0.219 & 0.225 \\
0.5 & 0.534 & 0.594 & 0.5 & 0.739 & 0.959 & 0.5 & 0.618 & 0.664 \\
1 & 1.010 & 1.035 & 0.5 & 0.601 & 0.693 & 0.5 & 0.565 & 0.594 \\
2 & 1.997 & 2.002 & 0.5 & 0.541 & 0.581 & 0.5 & 0.535 & 0.552 \\
5 & 4.993 & 4.989 & 0.5 & 0.511 & 0.521 & 0.5 & 0.513 & 0.520 \\
10 & 9.994 & 9.990 & 0.5 & 0.504 & 0.507 & 0.5 & 0.505 & 0.508 \\
0.5 & 0.584 & 0.752 & 2 & 2.467 & 2.861 & 0.5 & 0.647 & 0.711 \\
1 & 1.059 & 1.168 & 2 & 2.257 & 2.462 & 0.5 & 0.592 & 0.633 \\
2 & 2.042 & 2.123 & 2 & 2.163 & 2.297 & 0.5 & 0.562 & 0.590 \\
5 & 5.029 & 5.088 & 2 & 2.097 & 2.184 & 0.5 & 0.541 & 0.559 \\
10 & 10.024 & 10.073 & 2 & 2.066 & 2.129 & 0.5 & 0.530 & 0.543 \\
0.5 & 0.564 & 0.643 & 0.5 & 0.741 & 0.917 & 0.8 & 0.817 & 0.836 \\
1 & 1.035 & 1.081 & 0.5 & 0.612 & 0.686 & 0.8 & 0.805 & 0.816 \\
2 & 2.014 & 2.036 & 0.5 & 0.553 & 0.592 & 0.8 & 0.804 & 0.811 \\
5 & 5.001 & 5.005 & 0.5 & 0.517 & 0.533 & 0.8 & 0.803 & 0.807 \\
10 & 9.999 & 9.999 & 0.5 & 0.507 & 0.513 & 0.8 & 0.802 & 0.804 \\
0.5 & 0.638 & 0.835 & 2 & 2.348 & 2.586 & 0.8 & 0.835 & 0.860 \\
1 & 1.121 & 1.278 & 2 & 2.194 & 2.303 & 0.8 & 0.819 & 0.835 \\
2 & 2.111 & 2.249 & 2 & 2.137 & 2.212 & 0.8 & 0.813 & 0.823 \\
5 & 5.108 & 5.236 & 2 & 2.101 & 2.165 & 0.8 & 0.811 & 0.818 \\
10 & 10.118 & 10.250 & 2 & 2.081 & 2.139 & 0.8 & 0.810 & 0.816 \\
\hline & & & & & & & & \\
\hline & & 0.519 &
\end{tabular}

Table 2: Limiting DTMLE for MVN copula-based models with NB2 $(\mu ; \gamma)$ margins. The truncation point is chosen to exceed 0.999 for total probabilities.

likelihood in (5); because these are limits as $n \rightarrow \infty$ of $n^{-1}$ times the log-likelihood, $H$ is the inverse Fisher information, or equivalently, the covariance matrix for sample size $n$ is approximately $n^{-1} H$. For a comparison, we have also calculated the Hessian at the limit for the standard MLE. For simpler comparisons, we convert to standard errors (SE), say for a sample size of $n=100$ (that is, square roots of the diagonals of the above matrices divided by $n$ ). Some representative results are given in Table 3 for MVN copula-based 
models with Poisson margins, with the MSLE results omitted because they were again identical with MLE up to three or four decimal places. The results in Table 3 show that the surrogate likelihood method slightly underestimates the SEs and underestimation of the curvature increases as the dimension $d$ and/or the latent correlation $\rho$ increases when the DTMLE are correct.

\begin{tabular}{cc|cccc|cccc}
\hline$\mu$ & $\rho$ & \multicolumn{4}{|c|}{$\mathrm{SE}(\mu)$} & \multicolumn{4}{c}{$\mathrm{SE}(\rho)$} \\
& & \multicolumn{2}{c|}{$d=2$} & \multicolumn{2}{c}{$d=3$} & \multicolumn{2}{c}{$d=2$} & \multicolumn{2}{c}{$d=3$} \\
& & $\mathrm{ML}$ & $\mathrm{SUL}$ & $\mathrm{ML}$ & $\mathrm{DT}$ & $\mathrm{ML}$ & $\mathrm{DT}$ & $\mathrm{ML}$ & $\mathrm{DT}$ \\
\hline 0.5 & 0.2 & 0.054 & 0.055 & 0.046 & 0.050 & 0.135 & 0.133 & 0.087 & 0.088 \\
1 & 0.2 & 0.076 & 0.077 & 0.067 & 0.067 & 0.112 & 0.120 & 0.073 & 0.082 \\
2 & 0.2 & 0.109 & 0.109 & 0.096 & 0.096 & 0.101 & 0.105 & 0.067 & 0.071 \\
5 & 0.2 & 0.173 & 0.173 & 0.151 & 0.152 & 0.096 & 0.097 & 0.064 & 0.065 \\
10 & 0.2 & 0.244 & 0.245 & 0.217 & 0.215 & 0.095 & 0.096 & 0.063 & 0.063 \\
0.5 & 0.5 & 0.059 & 0.059 & 0.055 & 0.055 & 0.105 & 0.070 & 0.074 & 0.047 \\
1 & 0.5 & 0.085 & 0.084 & 0.079 & 0.077 & 0.086 & 0.071 & 0.061 & 0.049 \\
2 & 0.5 & 0.121 & 0.120 & 0.114 & 0.111 & 0.075 & 0.070 & 0.053 & 0.049 \\
5 & 0.5 & 0.193 & 0.192 & 0.181 & 0.180 & 0.070 & 0.068 & 0.049 & 0.048 \\
10 & 0.5 & 0.273 & 0.273 & 0.257 & 0.256 & 0.068 & 0.068 & 0.048 & 0.047 \\
0.5 & 0.8 & 0.065 & 0.063 & 0.063 & 0.059 & 0.055 & 0.032 & 0.040 & 0.022 \\
1 & 0.8 & 0.093 & 0.089 & 0.091 & 0.084 & 0.043 & 0.032 & 0.031 & 0.023 \\
2 & 0.8 & 0.133 & 0.129 & 0.130 & 0.123 & 0.036 & 0.031 & 0.025 & 0.022 \\
5 & 0.8 & 0.211 & 0.209 & 0.207 & 0.202 & 0.031 & 0.029 & 0.022 & 0.021 \\
10 & 0.8 & 0.317 & 0.298 & 0.294 & 0.290 & 0.031 & 0.029 & 0.021 & 0.020 \\
\hline
\end{tabular}

Table 3: Standard errors (SE) of the limiting DTMLE and MLE for MVN copula-based models with Poisson $(\mu)$ margins. The truncation point is chosen to exceed 0.999 for total probabilities.

\section{Small-sample efficiency based on simulation studies}

In this section we study the small-sample efficiency of the likelihood estimation methods in the case of discrete data with high-dimensional dependence. In particular we concentrate on modelling spatially aggregated (areal) binary and count data.

Let $G=(V, E)$ be the underlying graph, where $V=\{1,2, \ldots, d\}$ are the vertices and $E \subset V \times V$ are the edges of $G$. Each vertex of $\mathrm{G}$ corresponds to a region over which measurements have been aggregated, and each edge of $G$ represents the spatial adjacency of two such regions. Let $w_{j}$ be the degree of vertex $j$, let $\mathbf{D}=\operatorname{diag}\left(w_{1}, \ldots, w_{d}\right)$, let 
$\varrho \in[0,1)$, and let $\mathbf{A}=[1\{(i, j) \in E\}]$ be the adjacency matrix of $G$ with rows and columns labelled by graph vertices, with a 1 or 0 in position $(j, k)$ according to whether $j$ and $k$ are adjacent or not.

We adopt the same dependence model, the so named CAR copula, proposed by Hughes (2014). This is a MVN copula constructed by the inversion method in (1). In particular, if $\Phi_{d}(\cdot ; \Sigma)$ is the MVN cdf with covariance matrix

$$
\boldsymbol{\Sigma}=\left(\sigma_{j k}: 1 \leq j \leq k \leq d\right)
$$

and $N\left(0, \sigma_{j j}^{2}\right)$ margins, and $\Phi\left(\cdot ; \sigma_{j j}^{2}\right)$ 's are the univariate normal cdfs with variances $\sigma_{j j}^{2}$ 's, then the CAR copula is

$$
C\left(u_{1}, \ldots, u_{d} ; \Sigma\right)=\Phi_{d}\left(\Phi^{-1}\left(u_{1} ; \sigma_{11}^{2}\right), \ldots, \Phi^{-1}\left(u_{d}, \sigma_{d d}^{2}\right) ; \Sigma\right)
$$

where $\Sigma=(\mathbf{D}-\varrho \mathbf{A})^{-1}$ is the inverse of the precision matrix of the proper conditionally autoregressive (CAR) model (Gelfand and Vounatsou, 2003; Mardia, 1988). Hughes (2014) considered only Poisson $(\mu)$ margins. Here we also consider Bernoulli $(\mu), \operatorname{NB} 1(\mu, \gamma)$, and $\operatorname{NB} 2(\mu, \gamma)$ parametrization of the negative binomial distribution to allow for a comprehensive comparison. For the covariates we use the same design, i.e., we chose $p=2, \mathbf{x}_{j}=$ $\left(x_{1 j}, x_{2 j},\right)^{\top}$, where $x_{1 j}$ and $x_{2 j}$ are the coordinates of vertex $j$ and let $\mu$ depend on the covariates, that is $\nu_{j}=\eta\left(\mu_{j}\right)=\beta_{1} x_{1 j}+\beta_{2} x_{2 j}, j=1, \ldots, d$. For the regression parameters we use $\beta_{1}=-\beta_{2}=2$ for count and binary data, respectively. These regression coefficients lead to similar mean values with the ones used by Hughes (2014), but the size of the discrete probabilities increases. For the link function $\eta$, we took the log link function for Poisson and NB regression, and the logit link function or the probit link function for binary regression. Note also that binary and Poisson regression $\gamma$ is null, while for NB1 and NB2 regression $\gamma$ is scalar $(r=1)$.

We randomly generate data on the $15 \times 15$ square lattice, where the coordinates of the vertices were restricted to the unit square. We choose to focus on this lattice size since the Slovenia stomach cancer data (Zadnik and Reich, 2006) in the forthcoming section has a similar dimension. We also assume that we have a truly small sample that is just $n=1$ 
observation per lattice. For the CAR copula model and parameter and design selections we derive the estimates via the DT and SL method.

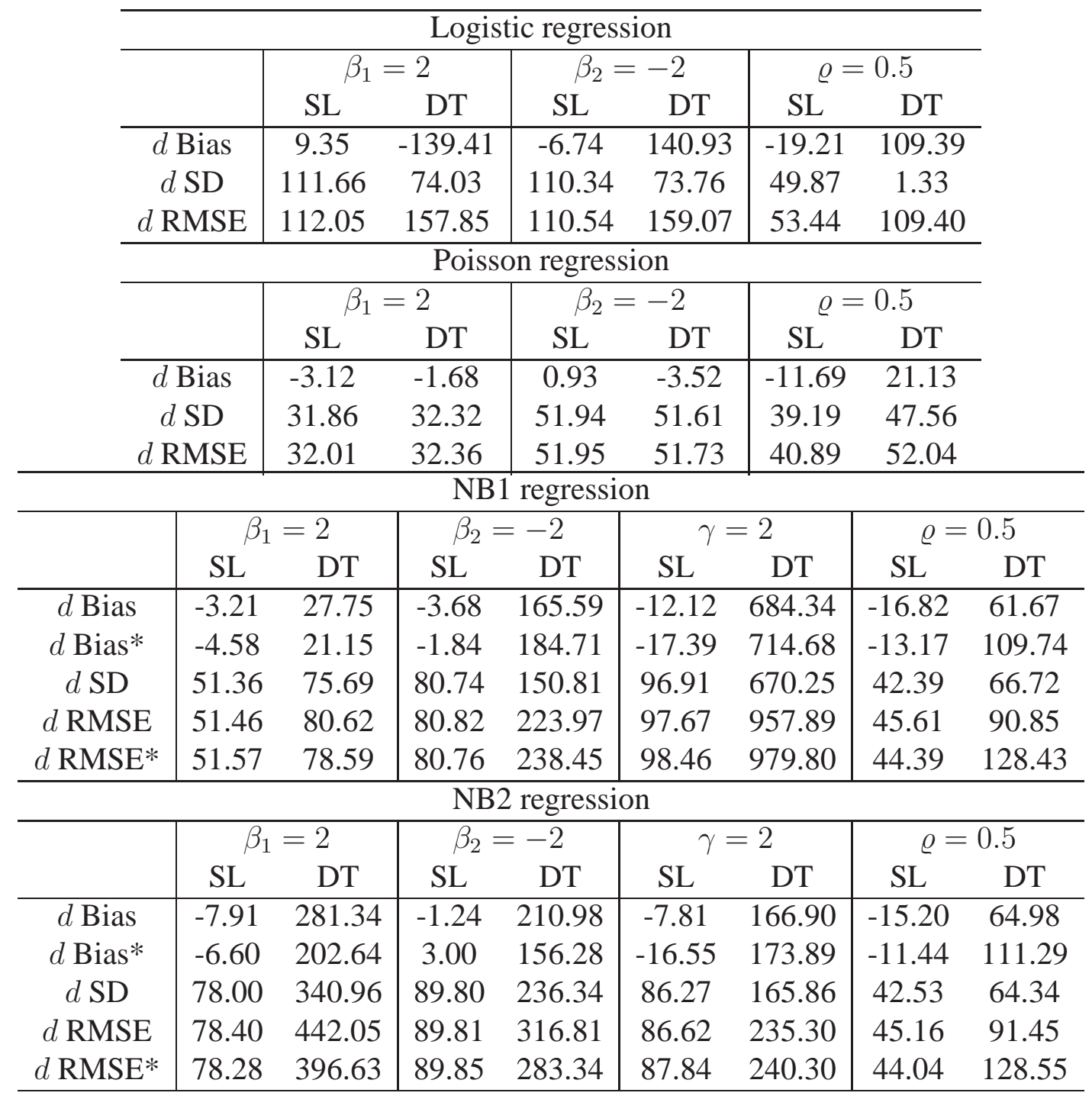

Table 4: The results of the simulation study for the $15 \times 15$ lattice and logistic, Poisson, NB1 and NB2 regression for spatially aggregated (areal) binary and count data for $\varrho=0.5$.

Representative summaries of findings on the performance of the approaches are given in Table 4 and Table 5 for $\varrho=0.5$ and $\varrho=0.8$, respectively. The tables contain the true parameter values, the bias, standard deviation (SD), and root mean square error (RMSE) scaled by $d=15^{2}$ of the DT and SL estimates from $10^{3}$ random samples generated from the CAR copula and marginal logistic, Poisson, NB1 and NB2 regression. For NB1 and NB2 regression in Table 4 the distribution of the DT estimators is quite skewed (e.g., $\hat{\varrho}$ 


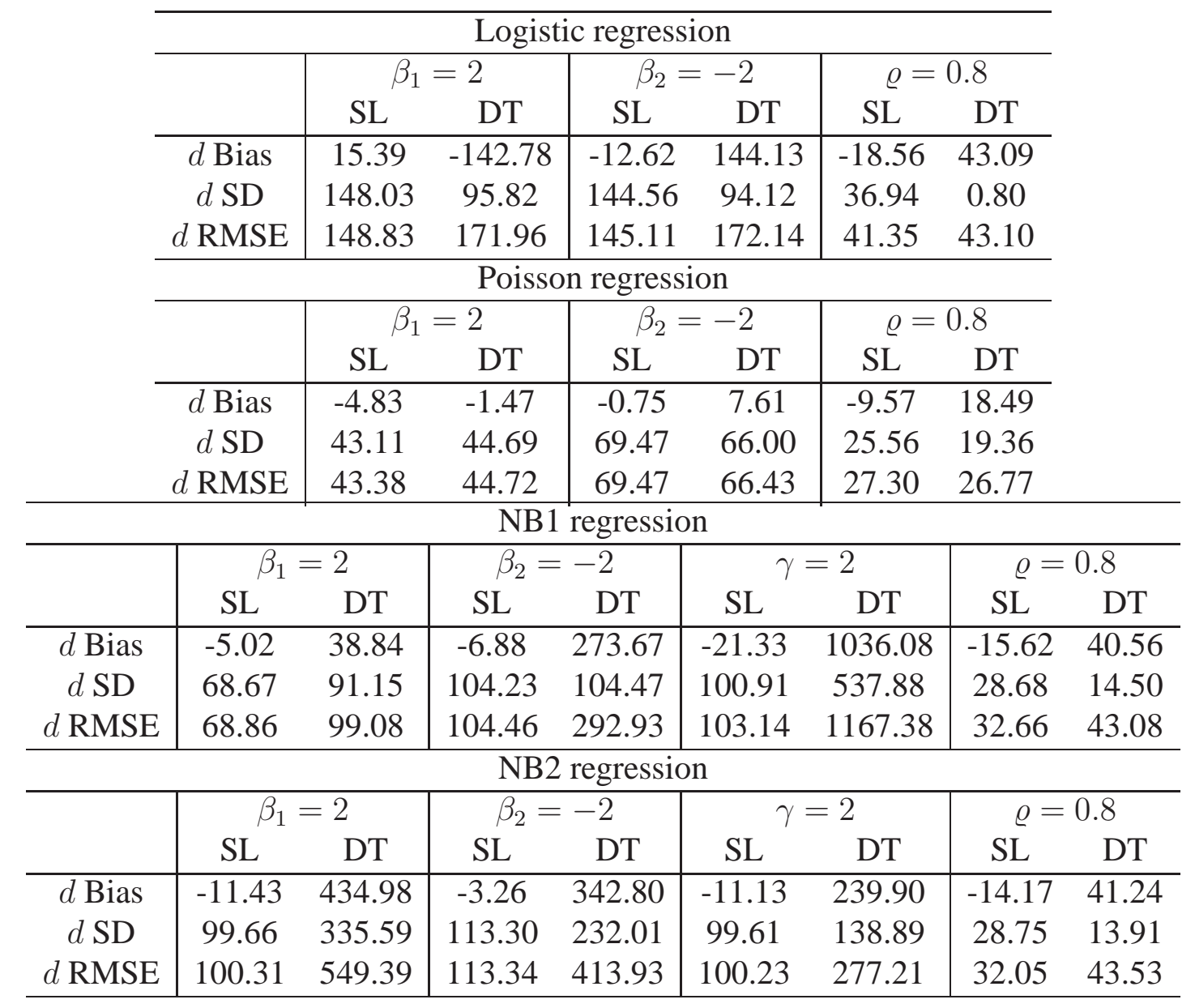

Table 5: The results of the simulation study for the $15 \times 15$ lattice and logistic, Poisson, NB1 and NB2 regression for spatially aggregated (areal) binary and count data for $\varrho=0.8$.

is skewed to the upper bound of the parameter space), thus we also calculate the sample median to be more informative. Asterisks indicate the corresponding biases and RMSEs.

Conclusions from the values in the tables are the following:

- The SL method is highly efficient according to the simulated biases and variances.

- The DT method yields estimates that are almost as good as the SL estimates for the regression parameters when there is less discretization (smaller individual probabilities).

- The DT method overestimates the univariate marginal parameters when there is more discretization (larger individual probabilities).

- The efficiency of the DT method is low for the parameter $\varrho$ of the CAR precision 
matrix. The parameter $\varrho$ is substantially overestimated and overestimation decreases as $\varrho$ increases or the individual probabilities decrease.

\section{Illustrations}

In this section we illustrate the methods with spatially aggregated count data. In the first subsection we apply the likelihood estimation methods to the Slovenia stomach cancer data (Zadnik and Reich, 2006), also analysed in Hughes (2014). In this areal dataset, there are small individual probabilities. In the second subsection the methods are in contrast applied to the Ohio lung cancer incidence data (Xia and Carlin, 1998) for which there apparently exist large individual probabilities. As emphasized in the preceding sections, the size of the discrete probabilities can substantially influence the efficiency of the surrogate likelihood method based on the DT. In fact in this section we also calculate/plot some simple descriptive statistics to form as diagnostics for the efficiency of DT method for the data on hand.

Model selection is often based on information criteria such as AIC, BIC, SBC or Generalized AIC in order to include a penalty for the different number of parameters among the models. We adopt one of this criteria, namely the AIC, here, since the NB regression with spatial discrete data has an additional parameter. The discussion below could also apply to other information criteria. By using the DT or SL method, the AIC is $-2 \times \log$-likelihood $+2 \times(\#$ model parameters) and a smaller AIC value indicates a better fitting model.

\subsection{The Slovenia data}

In this section we re-analyse the Slovenia stomach cancer incidence data in Hughes (2014). The Slovenia cancer incidence data consist of municipality-level $(d=194)$ observed deaths from stomach cancer in Slovenia for the period 1995-2001 (Zadnik and Reich, 2006) and are provided at the supplementary material in Hodges (2013). Number of expected deaths from stomach cancer and municipality specific socio-economic statuses as determined by Slovenia's Institute of Macroeconomic Analysis and Development are also available. The interest of this analysis is to explain the relationship of the stomach cancer cases as a 
function of the municipality specific socio-economic statuses.

The observed stomach cancer cases $y_{j}, j=1, \ldots, 194$ are counts, so we can assume a marginal Poisson or NB1 or NB2 model with means:

$$
\mu_{j}=\mathrm{E}_{j} \exp \left(\beta_{0}+\beta_{1} \operatorname{SoEc}_{j}\right), j=1, \ldots, 194
$$

where $\mathrm{E}_{j}$ and $\mathrm{SoEc}_{j}$ is the expected number of cases and standardised socio-economic status respectively for municipality $j$. For a preliminary analysis, we fit the model ignoring the spatial dependence, that is we assume independence. Figure 1 depicts the size of the estimated discrete probabilities for all models under the independence assumption. As revealed there are some small individual probabilities, suggesting that the surrogate likelihood method based on the DT might be reliable.

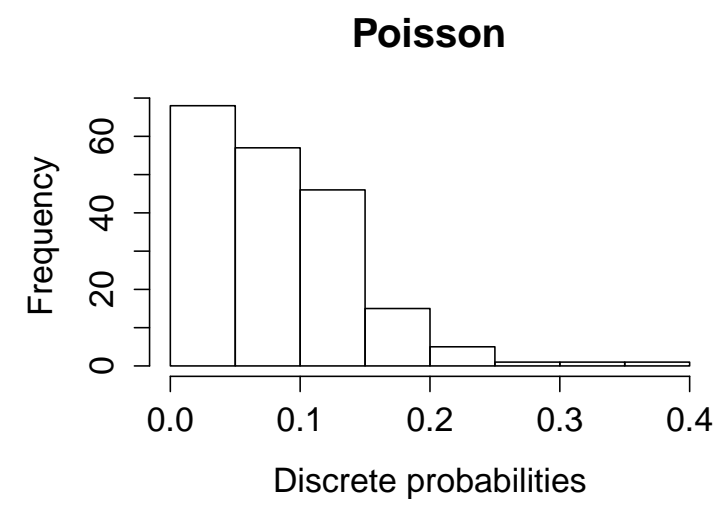

NB1

NB2
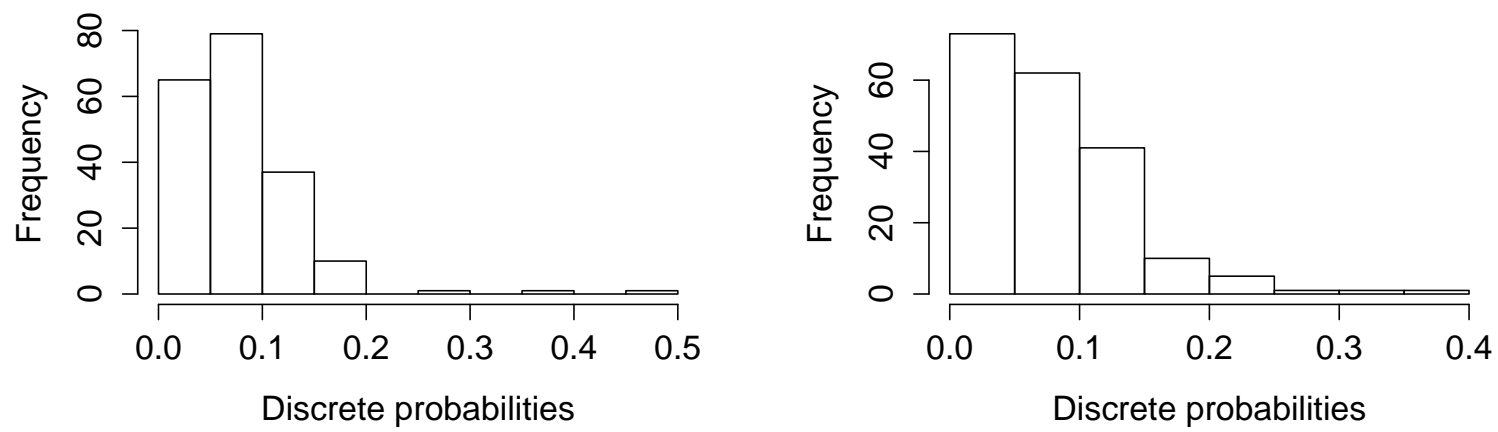

Figure 1: The size of the the discrete probabilities assuming independence for the Slovenia stomach cancer data.

Then, we fit the discrete CAR copula models performing estimation via the DT and SL 
methods. Table 6 gives the estimated parameters along with the AIC values. The best fit in terms of the penalized log-likelihood principle (AIC) is based on the MVN copula with an NB1 margin, where there is an improvement over the MVN copula with a Poisson. The AIC values show that NB1 regression is marginally better than NB2 regression, and both are far better than Poisson regression (Hughes, 2014). The estimate of the parameter $\varrho$ of the CAR precision matrix is significantly underestimated under the assumption of a Poisson margin; there is enough improvement to change a value of $\hat{\varrho}=0.29$ to one of $\hat{\varrho}=0.44$.

\begin{tabular}{c|cc|cc|cc}
\hline & \multicolumn{2}{|c|}{ Poisson regression } & \multicolumn{2}{|c|}{ NB1 regression } & \multicolumn{2}{c}{ NB2 regression } \\
& DT & SL & DT & SL & DT & SL \\
\hline$\beta_{0}$ & 0.153 & 0.153 & 0.144 & 0.145 & 0.144 & 0.145 \\
$\beta_{1}$ & -0.128 & -0.128 & -0.120 & -0.120 & -0.098 & -0.098 \\
$\gamma$ & - & - & 0.893 & 0.891 & 0.047 & 0.047 \\
$\varrho$ & 0.283 & 0.289 & 0.438 & 0.438 & 0.436 & 0.436 \\
\hline AIC & 1150.7 & 1150.4 & 1110.6 & 1110.4 & 1115.2 & 1115.0 \\
\hline
\end{tabular}

Table 6: Estimated parameters and AIC values using the DT and SL methods for the Slovenia stomach cancer data.
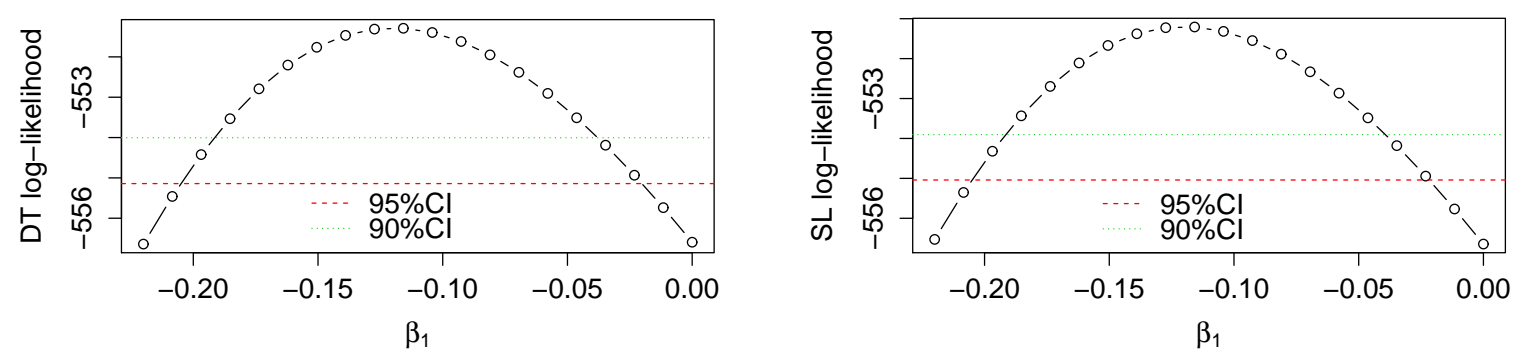

Figure 2: Profile likelihoods for the SoEc coefficient for the MVN copula with NB1 margins model fitted to the Slovenia stomach cancer data. The dashed and dotted horizontal lines indicate the $95 \%$ and $90 \%$ confidence intervals, respectively.

The estimated regression coefficient evidences a negative effect of SoEc with the number of cases. A further confirmation of the effect between cases and SoEc is given by the log-profile likelihoods of the SoEc coefficient displayed in Figure 2. The profile CIs show the SoEc is significantly negatively associated with excess of stomach cancer incidence. Our analysis also implies that the socio-economic status does not account for all of the 
spatial confounding in the count response since the estimate of $\varrho$ was as large as 0.44 .

\subsection{The Ohio data}

The Ohio lung cancer data consist of county-level $(d=88)$ deaths from lung cancer in Ohio for the period 1968-1988 (Xia and Carlin, 1998) and are provided at the supplementary material in Banerjee et al. (2014). They are stratified on race (whites vs non-whites) and gender (males vs females); that is there are $n=4$ observations available per county. The interest of this analysis is to explain the relationship of the lung cancer cases as a function of the stratified covariates. Another question of interest was whether the difference between males and females was different for whites and non-whites. Here we mainly illustrate the methods for the 1975 data only but the analysis can reproduced for the other years as well.

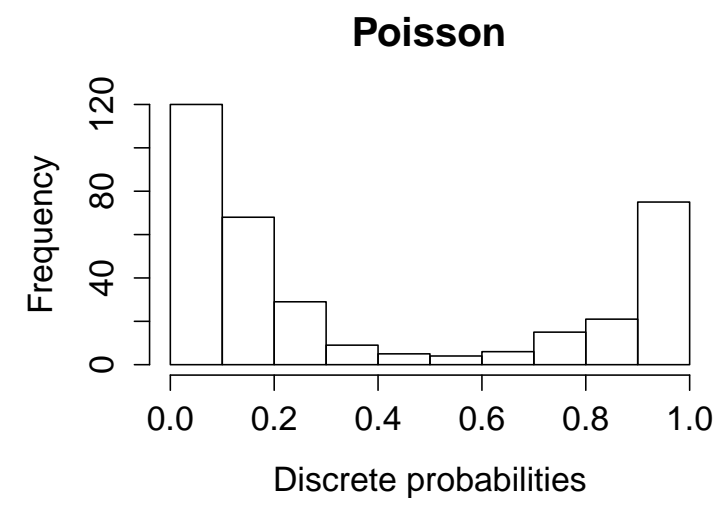

NB1

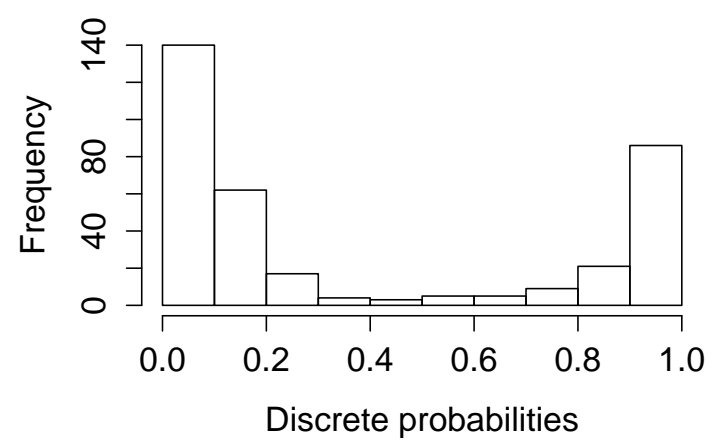

NB2

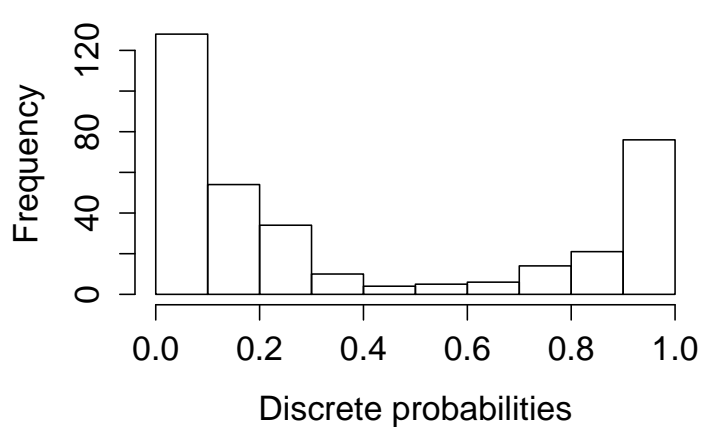

Figure 3: The size of the the discrete probabilities assuming independence for the Ohio lung cancer data.

The observed lung cancer cases $y_{i j}, i=1, \ldots, 4, j=1, \ldots, 88$ are counts, so we can 
assume a marginal Poisson or NB1 or NB2 model with means:

$\mu_{i j}=\mathrm{E}_{i j} \exp \left(\beta_{0}+\beta_{1}\right.$ Race $_{i j}+\beta_{2}$ Gender $\left._{i j}+\beta_{3} \operatorname{Race}_{i j} \times \operatorname{Gender}_{i j}\right), i=1, \ldots, 4, j=1, \ldots, 88$,

where $\mathrm{E}_{i j}$ is the expected number of cases. For a preliminary analysis, we fit the model ignoring the spatial dependence, that is we assume independence. Figure 3 depicts the size of the estimated discrete probabilities for all models under the independence assumption. As revealed there are some large individual probabilities, suggesting that the surrogate likelihood method based on the DT might not be reliable.

\begin{tabular}{|c|c|c|c|c|c|c|c|c|}
\hline & \multicolumn{8}{|c|}{ Poisson regression } \\
\hline & \multicolumn{4}{|c|}{ DT } & \multicolumn{4}{|c|}{ SL } \\
\hline & Est. & SE & $Z$ & $p$-value & Est. & SE & $Z$ & $p$-value \\
\hline$\overline{\beta_{0}}$ & -7.331 & 0.029 & -254.534 & $<0.001$ & -7.345 & 0.025 & -294.174 & $<0.001$ \\
\hline$\beta_{1}$ & 0.060 & 0.062 & 0.976 & 0.329 & 0.077 & 0.061 & 1.262 & 0.207 \\
\hline$\beta_{2}$ & -1.180 & 0.049 & -24.148 & $<0.001$ & -1.189 & 0.045 & -26.321 & $<0.001$ \\
\hline$\beta_{3}$ & -0.275 & 0.132 & -2.076 & 0.038 & -0.261 & 0.133 & -1.960 & 0.050 \\
\hline$\varrho$ & 0.540 & 0.166 & 3.247 & 0.001 & 0.385 & 0.147 & 2.626 & 0.009 \\
\hline \multirow[t]{4}{*}{ AIC } & \multicolumn{4}{|c|}{1255.6} & \multicolumn{4}{|c|}{1262.1} \\
\hline & \multicolumn{8}{|c|}{ NB1 regression } \\
\hline & \multicolumn{4}{|c|}{ DT } & \multicolumn{4}{|c|}{$\overline{\mathrm{SL}}$} \\
\hline & Est. & SE & $Z$ & $p$-value & Est. & SE & Z & $p$-value \\
\hline$\beta_{0}$ & -7.233 & 0.040 & -182.519 & $<0.001$ & -7.343 & 0.028 & -260.013 & $<0.001$ \\
\hline$\beta_{1}$ & 0.037 & 0.079 & 0.469 & 0.639 & 0.085 & 0.076 & 1.107 & 0.268 \\
\hline$\beta_{2}$ & -1.079 & 0.078 & -13.806 & $<0.001$ & -1.185 & 0.019 & -61.184 & $<0.001$ \\
\hline$\beta_{3}$ & -0.242 & 0.154 & -1.578 & 0.115 & -0.253 & 0.158 & -1.602 & 0.109 \\
\hline$\gamma$ & 2.452 & 0.488 & 5.028 & $<0.001$ & 0.596 & 0.227 & 2.621 & 0.009 \\
\hline$\varrho$ & 0.989 & 0.003 & 324.739 & $<0.001$ & 0.448 & 0.015 & 29.461 & $<0.001$ \\
\hline \multirow[t]{4}{*}{ AIC } & \multicolumn{4}{|c|}{1197.644} & \multicolumn{4}{|c|}{1240.162} \\
\hline & \multicolumn{8}{|c|}{ NB2 regression } \\
\hline & \multicolumn{4}{|c|}{$\overline{\mathrm{DT}}$} & \multicolumn{4}{|c|}{ SL } \\
\hline & Est. & SE & $Z$ & $p$-value & Est. & SE & $Z$ & $p$-value \\
\hline$\beta_{0}$ & -7.369 & 0.054 & -137.178 & $<0.001$ & -7.386 & 0.037 & -202.052 & $<0.001$ \\
\hline$\beta_{1}$ & 0.087 & 0.096 & 0.908 & 0.364 & 0.096 & 0.088 & 1.088 & 0.277 \\
\hline$\beta_{2}$ & -1.209 & 0.086 & -14.104 & $<0.001$ & -1.237 & 0.066 & -18.735 & $<0.001$ \\
\hline$\beta_{3}$ & -0.247 & 0.174 & -1.417 & 0.156 & -0.219 & 0.166 & -1.322 & 0.186 \\
\hline$\gamma$ & 0.032 & 0.012 & 2.681 & 0.007 & 0.029 & 0.010 & 3.015 & 0.003 \\
\hline$\varrho$ & 0.675 & 0.266 & 2.537 & 0.011 & 0.331 & 0.183 & 1.807 & 0.071 \\
\hline AIC & \multicolumn{4}{|c|}{1225.601} & \multicolumn{4}{|c|}{1232.910} \\
\hline
\end{tabular}

Table 7: Estimated parameters, standard errors (SE) and AIC values using the DT and SL methods for the Ohio lung cancer data for the 1975 data. 
Then, we fit the discrete CAR copula models performing estimation via the DT and SL methods. Table 7 gives the estimated parameters and their standard errors (SE) along with the AIC values. The SEs of the maximum SL and DT estimates are obtained via the gradients and the Hessian computed numerically during the maximization process. Assuming that the usual regularity conditions (Serfling, 1980) for asymptotic maximum likelihood theory hold for the bivariate model as well as for its margins we have that the estimates are asymptotically normal. Therefore we also build and present Wald tests to statistically judge the effect of any covariate. The best fit in terms of the penalized log-likelihood principle (AIC) is based on the MVN copula with an NB2 margin, where there is an improvement over the MVN copula with a Poisson. The AIC values show that NB2 regression is marginally better than NB1 regression, and both are far better than Poisson regression.

Because there is much discretization in the data, the dispersion parameter $\gamma$ and parameter $\varrho$ of the CAR precision matrix are (substantially under the assumption of an NB1 margin) over-estimated. This was expected for overdispersed count data as shown in the studies of the properties of the surrogate likelihood estimates in Section 4 and Section 5.

In particular for areal data applications an interesting advantage of the CAR copula modelling over the classic areal GLM modelling is the ability to recover $\varrho$; see Hughes (2014) and the references therein. So an efficient estimation of $\varrho$ is highly desirable in this context; hence the DT method performs poorly in this example.

Based on our analysis, the SEs show the gender effect to be highly significant, and the gender by race interaction insignificant. However, for the DT analysis with Poisson regression, the gender by race interaction is statistically significant. Generally speaking, this implies that misspecifying the model or using the surrogate likelihood method based on the DT could lead to invalid conclusions.

The latter was actually the case for the analysis of the data for year 1974 in Table 8. The DT method resulted a statical significant ( $p$-value $=0.041$ ) rather than the true marginal statistical significant $(p$-value $=0.071)$ gender by race interaction under the best model (NB2) via the penalized likelihood principle (AIC). 


\begin{tabular}{|c|c|c|c|c|c|c|c|c|}
\hline & \multicolumn{8}{|c|}{ Poisson regression } \\
\hline & \multicolumn{4}{|c|}{ DT } & \multicolumn{4}{|c|}{ SL } \\
\hline & Est. & SE & $Z$ & $p$-value & Est. & SE & $Z$ & $p$-value \\
\hline$\beta_{0}$ & -7.429 & 0.024 & -308.975 & $<0.001$ & -7.429 & 0.024 & -311.736 & $<0.001$ \\
\hline$\beta_{1}$ & 0.134 & 0.060 & 2.250 & 0.024 & 0.137 & 0.059 & 2.315 & 0.021 \\
\hline$\beta_{2}$ & -1.276 & 0.043 & -29.854 & $<0.001$ & -1.276 & 0.041 & -31.326 & $<0.001$ \\
\hline$\beta_{3}$ & -0.385 & 0.140 & -2.745 & 0.006 & -0.382 & 0.140 & -2.724 & 0.006 \\
\hline$\varrho$ & 0.144 & 0.191 & 0.752 & 0.452 & 0.148 & 0.181 & 0.816 & 0.415 \\
\hline \multirow[t]{4}{*}{ AIC } & \multicolumn{4}{|c|}{1250.759} & \multicolumn{4}{|c|}{1255.702} \\
\hline & \multicolumn{8}{|c|}{ NB1 regression } \\
\hline & \multicolumn{4}{|c|}{ DT } & \multicolumn{4}{|c|}{ SL } \\
\hline & Est. & $\mathrm{SE}$ & $Z$ & $p$-value & Est. & SE & Z & $p$-value \\
\hline$\beta_{0}$ & -7.257 & 0.042 & -173.526 & $<0.001$ & -7.434 & 0.030 & -244.893 & $<0.001$ \\
\hline$\beta_{1}$ & 0.019 & 0.084 & 0.226 & 0.821 & 0.151 & 0.075 & 2.017 & 0.044 \\
\hline$\beta_{2}$ & -1.190 & 0.083 & -14.372 & $<0.001$ & -1.266 & 0.053 & -23.698 & $<0.001$ \\
\hline$\beta_{3}$ & -0.272 & 0.173 & -1.576 & 0.115 & -0.362 & 0.172 & -2.105 & 0.035 \\
\hline$\gamma$ & 1.857 & 0.456 & 4.068 & $<0.001$ & 0.608 & 0.161 & 3.771 & $<0.001$ \\
\hline$\varrho$ & 0.976 & 0.012 & 83.868 & $<0.001$ & 0.145 & 0.259 & 0.558 & 0.577 \\
\hline \multirow[t]{4}{*}{ AIC } & \multicolumn{4}{|c|}{1218.693} & \multicolumn{4}{|c|}{1231.642} \\
\hline & \multicolumn{8}{|c|}{ NB2 regression } \\
\hline & \multicolumn{4}{|c|}{ DT } & \multicolumn{4}{|c|}{ SL } \\
\hline & Est. & SE & $Z$ & $p$-value & Est. & SE & $Z$ & $p$-value \\
\hline$\beta_{0}$ & -7.487 & 0.043 & -173.800 & $<0.001$ & -7.491 & 0.039 & -191.327 & $<0.001$ \\
\hline$\beta_{1}$ & 0.156 & 0.092 & 1.696 & 0.090 & 0.169 & 0.090 & 1.886 & 0.059 \\
\hline$\beta_{2}$ & -1.251 & 0.070 & -17.825 & $<0.001$ & -1.254 & 0.117 & -10.710 & $<0.001$ \\
\hline$\beta_{3}$ & -0.365 & 0.179 & -2.041 & 0.041 & -0.364 & 0.202 & -1.804 & 0.071 \\
\hline$\gamma$ & 0.035 & 0.011 & 3.075 & 0.002 & 0.033 & 0.010 & 3.186 & 0.001 \\
\hline$\varrho$ & 0.401 & 0.290 & 1.380 & 0.168 & 0.288 & 0.261 & 1.106 & 0.269 \\
\hline AIC & \multicolumn{4}{|c|}{1200.528} & \multicolumn{4}{|c|}{1206.163} \\
\hline
\end{tabular}

Table 8: Estimated parameters, standard errors (SE) and AIC values using the DT and SL methods for the Ohio lung cancer data for the 1974 data.

\section{Discussion}

In this paper we have studied high-dimensional MVN copula models with discrete margins for analysing spatial discrete response data. We discussed simulated and surrogate (based on the DT) likelihood estimation methods. For the binary, Poisson, and negative binomial regression models with the MVN/CAR copula, we have shown that the surrogate likelihood method based on the DT leads to substantial upward bias for the estimates of the latent correlation/parameter of the precision matrix of the CAR model and the univariate marginal 
parameters when there is more discretization, that is large individual discrete probabilities.

We have shown that the SL method proposed by Nikoloulopoulos (2013b), is highly efficient for a high-dimensional discrete response up to dimension $d=15^{2}$. Although there is an issue of computational burden as the dimension and the sample size increase, this will subside, as computing technology is advancing rapidly. Any comparison of the methods in terms of computing time is a digression. It is obvious that the DT method is much faster then the SL method, since a numerically more difficult high-dimensional MVN rectangle probability calculation is replaced with a much simpler computationally MVN density value. However, theoretically there are still problems for large individual probabilities, since the DT approximation of 'large steps' in the cdf is poor. In fact, we novelty propose simple diagnostics (descriptive statistics such as a histogram) to judge if the DT method is reliable and reduce by its use the computational burden when it is possible (i.e., for small individual probabilities).

It is worth mentioning that the range of possible applications of these tools goes beyond biometric/disease/health data and is of interest also in other fields such as hydrometeorology. A typical example are the binary vectors describing the rainfall occurrence at multiple sites, or the occurrence of simultaneous exceedance of given threshold values in extreme value analysis of floods, droughts, and storms over specified areas. Note also in passing the DT method deteriorates for such (binary) response data.

Finally, the results will be similar for other structured latent correlation structures such as the Matérn isotropic structure used for example in Madsen (2009) and Kazianka (2013). As previously emphasized the idea of the DT transform is to replace a numerically more difficult MVN rectangle probability calculation with a simpler MVN density value, and hence it is discrete responses that matter and not the type of the structured correlation structure.

\section{References}

Aitchinson, J. and Ho, C. (1989). The multivariate Poisson-log normal distribution. Biometrika, 75:621-629. 
Banerjee, S., Carlin, B. P., and Gelfand, A. E. (2014). Hierarchical Modeling and Analysis for Spatial Data. Chapman \& Hall, London.

Cameron, A. C. and Trivedi, P. K. (1998). Regression Analysis of Count Data. Cambridge University Press.

Denuit, M. and Lambert, P. (2005). Constraints on concordance measures in bivariate discrete data. Journal of Multivariate Analysis, 93(1):40-57.

Diggle, P. and Ribeiro, P. J. (2007). Model-based Geostatistics. Springer.

Diggle, P. J., Tawn, J. A., and Moyeed, R. A. (1998). Model-based geostatistics. Journal of the Royal Statistical Society: Series C (Applied Statistics), 47(3):299-350.

Ferguson, T. S. (1967). Mathematical Statistics: A Decision Theoretic Approach. Probability and Mathematical Statistics, Vol. 1. Academic Press, New York.

Gelfand, A. E. and Vounatsou, P. (2003). Proper multivariate conditional autoregressive models for spatial data analysis. Biostatistics, 4(1):11-15.

Genz, A. (1992). Numerical computation of the multivariate normal probabilities. Journal of Computational and Graphical Statistics, 1:141-150.

Genz, A. and Bretz, F. (2002). Methods for the computation of the multivariate $t$ probabilities. Journal of Computational and Graphical Statistics, 11:950-971.

Genz, A. and Bretz, F. (2009). Computation of Multivariate Normal and t Probabilities. Springer.

Genz, A., Bretz, F., Miwa, T., Mi, X., Leisch, F., Scheipl, F., and Hothorn, T. (2012). mvtnorm: Multivariate Normal and t Distributions. R package version 0.9-9992.

Hodges, J. (2013). Richly Parameterized Linear Models: Additive, Time Series, and Spatial Models Using Random Effects. Chapman \& Hall, London.

Hughes, J. (2014). copCAR: A flexible regression model for areal data. Journal of Computational and Graphical Statistics. http://dx.doi.org/10.1080/10618600.2014.948178.

Joe, H. (1997). Multivariate Models and Dependence Concepts. Chapman \& Hall, London.

Joe, H. (2014). Dependence Modeling with Copulas. Chapman \& Hall, London. 
Johnson, N. L. and Kotz, S. (1972). Continuous Multivariate Distributions. Wiley, New York.

Kazianka, H. (2013). Approximate copula-based estimation and prediction of discrete spatial data. Stochastic Environmental Research and Risk Assessment, 27(8):2015-2026.

Kazianka, H. and Pilz, J. (2010). Copula-based geostatistical modeling of continuous and discrete data including covariates. Stochastic Environmental Research and Risk Assessment, 24(5):661-673.

Lawless, J. F. (1987). Negative binomial and mixed Poisson regression. The Canadian Journal of Statistics, 15(3):209-225.

Madsen, L. (2009). Maximum likelihood estimation of regression parameters with spatially dependent discrete data. Journal of Agricultural, Biological, and Environmental Statistics, 14:375-391.

Madsen, L. and Fang, Y. (2011). Joint regression analysis for discrete longitudinal data. Biometrics, 67:1171-1175.

Mardia, K. V. (1988). Multi-dimensional multivariate gaussian markov random fields with application to image processing. Journal of Multivariate Analysis, 24(2):265-284.

Nash, J. (1990). Compact Numerical Methods for Computers: Linear Algebra and Function Minimisation. Hilger, New York. 2nd edition.

Nelsen, R. B. (2006). An Introduction to Copulas. Springer-Verlag, New York.

Nikoloulopoulos, A. K. (2013a). Copula-based models for multivariate discrete response data. In Durante, F., Härdle, W., and Jaworski, P., editors, Copulae in Mathematical and Quantitative Finance, pages 231-249. Springer.

Nikoloulopoulos, A. K. (2013b). On the estimation of normal copula discrete regression models using the continuous extension and simulated likelihood. Journal of Statistical Planning and Inference, 143:1923-1937.

Nikoloulopoulos, A. K. and Joe, H. (2013). Factor copula models for item response data. Psychometrika. http://dx.doi.org/10.1007/s11336-013-9387-4. 
Nikoloulopoulos, A. K., Joe, H., and Chaganty, N. R. (2011). Weighted scores method for regression models with dependent data. Biostatistics, 12:653-665.

Rüschendorf, L. (1981). Stochastically ordered distributions and monotonicity of the ocfunction of sequential probability ratio tests. Series Statistics, 12(3):327-338.

Rüschendorf, L. (2009). On the distributional transform, sklar's theorem, and the empirical copula process. Journal of Statistical Planning and Inference, 139(11):3921-3927.

Serfling, R. J. (1980). Approximation Theorems of Mathematical Statistics. Wiley, New York.

Shi, P. and Valdez, E. A. (2014a). Longitudinal modeling of insurance claim counts using jitters. Scandinavian Actuarial Journal, 2014(2):159-179.

Shi, P. and Valdez, E. A. (2014b). Multivariate negative binomial models for insurance claim counts. Insurance: Mathematics and Economics, 55:18-29.

Sklar, M. (1959). Fonctions de répartition à $n$ dimensions et leurs marges. Publications de l'Institut de Statistique de l'Université de Paris, 8:229-231.

Song, P. X.-K. (2007). Correlated Data Analysis: Modeling, Analytics, and Application. Springer, NY.

Varin, C. (2008). On composite marginal likelihoods. Advances in Statistical Analysis, 92:1-28.

Varin, C., Reid, N., and Firth, D. (2011). An overview of composite likelihood methods. Statistica Sinica, 21:5-42.

Xia, H. and Carlin, B. P. (1998). Spatio-temporal models with errors in covariates: mapping Ohio lung cancer mortality. Statistics in Medicine, 17(18):2025-2043.

Zadnik, V. and Reich, B. (2006). Analysis of the relationship between socioeconomic factors and stomach cancer incidence in slovenia. Neoplasma, 53(2):103-110.

Zhao, Y. and Joe, H. (2005). Composite likelihood estimation in multivariate data analysis. The Canadian Journal of Statistics, 33(3):335-356. 